\title{
Protocolos clínicos em Hipomineralização Molar Incisivo (HMI): revisão integrativa
}

\section{da literatura}

\author{
Clinical protocols in Molar-Incisor Hypomineralization (MIH): an integrative literature review \\ Protocolos clínicos en la Hipomineralización Molar-Incisivo (HMI): una revisión bibliográfica
}

integradora

Recebido: 09/10/2021 | Revisado: 15/10/2021 | Aceito: 19/10/2021 | Publicado: 21/10/2021

\author{
Maria Clara de Oliveira Alves \\ ORCID: https://orcid.org/0000-0002-8969-0540 \\ Centro Universitário de Patos de Minas, Brasil \\ E-mail: mariaclara@unipam.edu.br \\ Bruna Stefany de Freitas Camargos \\ ORCID: https://orcid.org/0000-0003-1872-6195 \\ Centro Universitário de Patos de Minas, Brasil \\ E-mail: brunacamargos@unipam.edu.br \\ Thiago de Amorim Carvalho \\ ORCID: https://orcid.org/0000-0003-1153-0931 \\ Centro Universitário de Patos de Minas, Brasil \\ E-mail: thiagocarvalho@unipam.edu.br \\ Fabrício Campos Machado \\ ORCID: https://orcid.org/0000-0003-4603-8795 \\ Centro Universitário de Patos de Minas, Brasil \\ E-mail: fabriciocampos@unipam.edu.br
}

\begin{abstract}
Resumo
A hipomineralização molar-incisivo (HMI) é definida como um defeito qualitativo do esmalte caracterizada pelo aparecimento de lesões opacas de coloração branca, amarela ou marrom, que variam em tamanho e apresentam rápida progressão de lesões de cárie e hipersensibilidade, que afeta os molares e os incisivos. As modalidades de tratamento para os dentes afetados com essa condição são extensas, variam de acordo com o grau e localização da HMI tornando-se um desafio para os cirurgiões dentistas. O objetivo desse estudo é descrever os protocolos clínicos e os planos de cuidados para pessoas com hipomineralização molar-incisivo. Foi realizada uma revisão integrativa da literatura, utilizando as chaves de busca "Molar incisor hypomineralization" "Clinical Protocols" "Children" "Treatment", com o operador booleano "and", por meio da qual foram selecionados a partir dos critérios de elegibilidade propostos na estratégia PRISMA ScR, 10 artigos para análise qualitativa, nas bases de dados SciELO, Pubmed e BVs. Percebeu-se as modalidades de tratamento para os dentes afetados por HMI variam amplamente, dependendo da gravidade, e da presença de fatores complicadores, como hipersensibilidade, higiene oral e cooperação do paciente. Conclui-se, portanto, atualmente, não há consenso sobre a superioridade de tratamento de dentes afetados por hipomineralização molar-incisivo $(\mathrm{MIH})$, já que se tem o desafio das as fraturas pós eruptivas, hipersensibilidade dentinária e a dificuldade de adesão dentinária. Mais estudos são necessários para que este agravo comum na contemporaneidade clínica odontológica tenha de fato uma forma eficaz de ser solucionada.
\end{abstract}

Palavras-chave: Esmalte dental; Odontopediatria; Odontologia.

\begin{abstract}
Molar-incisor hypomineralization (MIH) is defined as a qualitative enamel defect characterized by the appearance of opaque white, yellow or brown lesions that vary in size and show rapid progression of caries lesions and hypersensitivity, affecting molars and incisors. Treatment modalities for teeth affected with this condition are extensive, varying according to the degree and location of MIH making it a challenge for dental surgeons. The aim of this study is to describe the clinical protocols and care plans for people with molar-incisor hypomineralization. An integrative literature review was carried out using the search keys "Molar incisor hypomineralization" "Clinical Protocols" "Children" "Treatment", with the Boolean operator "and", through which 10 articles were selected from the eligibility criteria proposed in the PRISMA ScR strategy for qualitative analysis, in the SciELO, Pubmed and BVs databases. It was realized that treatment modalities for teeth affected by MIH vary widely, depending on the severity, and the presence of complicating factors, such as hypersensitivity, oral hygiene, and patient cooperation. It is therefore concluded that there is currently no consensus on the superiority of treatment of teeth affected by molar-incisor hypomineralization (MIH), since there is the challenge of post-
\end{abstract}


eruptive fractures, dentin hypersensitivity and the difficulty of dentin adhesion. More studies are needed so that this common problem in contemporary clinical dentistry may have an effective way to be solved.

Keywords: Dental enamel; Pediatric dentistry; Dentistry.

\begin{abstract}
Resumen
La hipomineralización molar-incisiva (HMI) se define como un defecto cualitativo del esmalte que se caracteriza por la aparición de lesiones opacas de color blanco, amarillo o marrón que varían en tamaño y presentan una rápida progresión de las lesiones de caries e hipersensibilidad, afectando a molares e incisivos. Las modalidades de tratamiento de los dientes afectados por esta enfermedad son amplias y varían según el grado y la localización de la HMI, lo que supone un reto para los cirujanos dentales. El objetivo de este estudio es describir los protocolos clínicos y los planes de atención para las personas con hipomineralización molar-incisiva. Se realizó una revisión bibliográfica integradora utilizando las claves de búsqueda "Molar incisor hypomineralization" "Clinical Protocols" "Children" "Treatment", con el operador booleano "and", mediante la cual se seleccionaron 10 artículos a partir de los criterios de elegibilidad propuestos en la estrategia PRISMA ScR para el análisis cualitativo, en las bases de datos SciELO, Pubmed y BVs. Se ha comprobado que las modalidades de tratamiento de los dientes afectados por HMI varían mucho, dependiendo de la gravedad y de la presencia de factores de complicación, como la hipersensibilidad, la higiene bucal y la cooperación del paciente. Se concluye, por tanto, que actualmente no hay consenso sobre la superioridad del tratamiento de los dientes afectados por la hipomineralización molar-incisiva (HMI), ya que existe el reto de las fracturas post-eruptivas, la hipersensibilidad dentinaria y la dificultad de adhesión de la dentina. Se necesitan más estudios para que este agravio común en la odontología clínica contemporánea tenga una vía de solución efectiva.
\end{abstract}

Palabras clave: Esmalte dental; Odontología pediátrica; Odontología.

\title{
1. Introdução
}

Durante o desenvolvimento do esmalte podem surgir distúrbios denominados Defeitos de Desenvolvimento Do Esmalte (DEE) que geram, por exemplo, defeitos na translucidez e/ou cor do esmalte (Bezamat et al., 2021). Esses impactos no desenvolvimento do esmalte são críticos, visto que este tecido não tem capacidade de reparo celular, assim, não se remodela (Lacruz et al., 2017; Smith et al., 2017).

Esses defeitos podem apresentar como dois grupos: os que afetam a quantidade, chamados de hipoplasia de esmalte e aqueles que afetam a qualidade denominados hipomineralização do esmalte (Folayan et al., 2018). Dentre essas últimas alterações, destaca-se a Hipomineralização Molar- Incisivo (HMI), a qual apenas após o ano de 2001 no Congresso da Academia Europeia de Odontologia Pediátrica (EAPD), em Bergen, recebeu esse nome (Farias et al.,2018). A HMI é definida como defeito qualitativo do esmalte, que afeta de um a quatro primeiros molares permanentes e frequentemente afeta também os incisivos permanentes. (Folayan et al., 2018).

Os dentes acometidos por HMI, possuem propriedades mecânicas inferiores, como módulo de elasticidade reduzido, quando comparados aos dentes com esmalte sadio, em virtude de que, molecularmente, eles apresentam grande quantidade de proteínas, que inibem o crescimento de cristais de hidroxiapatita durante a maturação do esmalte (Bezamat et al., 2021). As características microestruturais do esmalte aumentam o risco de progressão rápida da doença cárie, que pode levar à perda do dente em idades muito precoces. A frequência de intervenções restauradoras e a necessidade de retratamento são significativamente maiores do que em grupos de pacientes que não apresentam essa patologia, de modo que o diagnóstico precoce é essencial para evitar consequências subsequentes. (Lopez Jordi et al., 2014).

A HMI é altamente prevalente em todo o mundo, afetando 878 milhões de pessoas, com 17,5 milhões de novos casos a cada ano (Schwendicke et al., 2018; Cunha et al., 2020). Ademais, a prevalência desse defeito de esmalte varia, mas tem sido consistentemente relatada entre $1 \%$ e $35 \%$ em todas as partes do mundo (Bezamat et al., 2021). Desse modo, as modalidades de tratamento para os dentes afetados com essa condição são extensas, variam de acordo com o grau e localização da HMI e vão desde a prevenção, restaurações e até mesmo a extração, tornando um desafio para os cirurgiões dentistas (Schwendicke et al., 2018; Silva et al., 2020). 
Existem várias opções de tratamento e a decisão terapêutica deve considerar a gravidade da doença, a idade do paciente, a capacidade cooperativa, status socioeconômico, a importância ortodôntica do dente afetado, a presença de outras anomalias menores e a expectativa do paciente. As crianças com defeitos de esmalte extensos geralmente requerem ações multidisciplinares realizadas por odontopediatras, clínicos gerais, protesistas, entre outros (Ochoa et al. 2017).

O objetivo desse estudo é descrever os protocolos clínicos e os planos de cuidados para pessoas com hipomineralização molar-incisivo.

\section{Metodologia}

Trata-se de uma revisão integrativa, adotando-se a estratégia PICO para a elaboração da pergunta norteadora, em que se atribuiu ao P (população) crianças com Hipomineralização Molar Incisivo (HMI), I (intervenção ou indicador) o tratamento da HMI e C (comparação) protocolos clínicos em crianças que não possuem Hipomineralização Molar Incisivo. O elemento O (outcome ou desfecho) referente aos protocolos clínicos adequados para o tratamento de HMI. Portanto a pergunta do estudo a ser respondida é: “quais os protocolos clínicos mais adequados para o tratamento de crianças com HMI?".

Essa revisão foi relatada seguindo o Guia PRISMA Scr, nas seguintes bases de dados: US National Library of Medicine National Institutes of Health (PubMed Central), Scientific Electronic Library Online (SciELO) e Bibblioteca Virtual em Saúde (BVS) utilizando-se os Descritores em Ciências da Saúde (DeCS): "Molar incisor hypomineralization" "Clinical Protocols" "Children" "Treatment". Para todas as buscas foram utilizados os mesmos descritores em inglês e português. Os estudos foram localizados utilizando ferramentas de pesquisa avançada, nas quais os termos da pesquisa serão cruzados e combinados com operador booleano "AND".

Como critérios de inclusão serão considerados: artigos com textos completos em língua inglesa e portuguesa, no período de publicação de 2016 a 2021. Foram excluídos trabalhos incluídos na literatura cinzenta, ou seja, sem revisão cega por pares tais como teses e dissertações, capítulos de livros, opiniões pessoais ou de especialistas, além de artigos fora do intervalo de tempo definido e em outro idioma, também indisponibilidade de texto completo.

\section{Resultados}

A busca de dados resultou em 108 artigos potencialmente elegíveis, dos quais 32 encontrados nas bases de dados US National Library of Medicine National Institutes of Health (PubMed Central), 13 artigos na Scientific Electronic Library Online (SciELO) e 63 artigos na Biblioteca Virtual em Saúde (BVS), e o fluxo de seleção está demonstrado na Figura 1. 
Figura 1. Fluxograma de seleção dos artigos, a partir da aplicação dos critérios de elegibilidade.
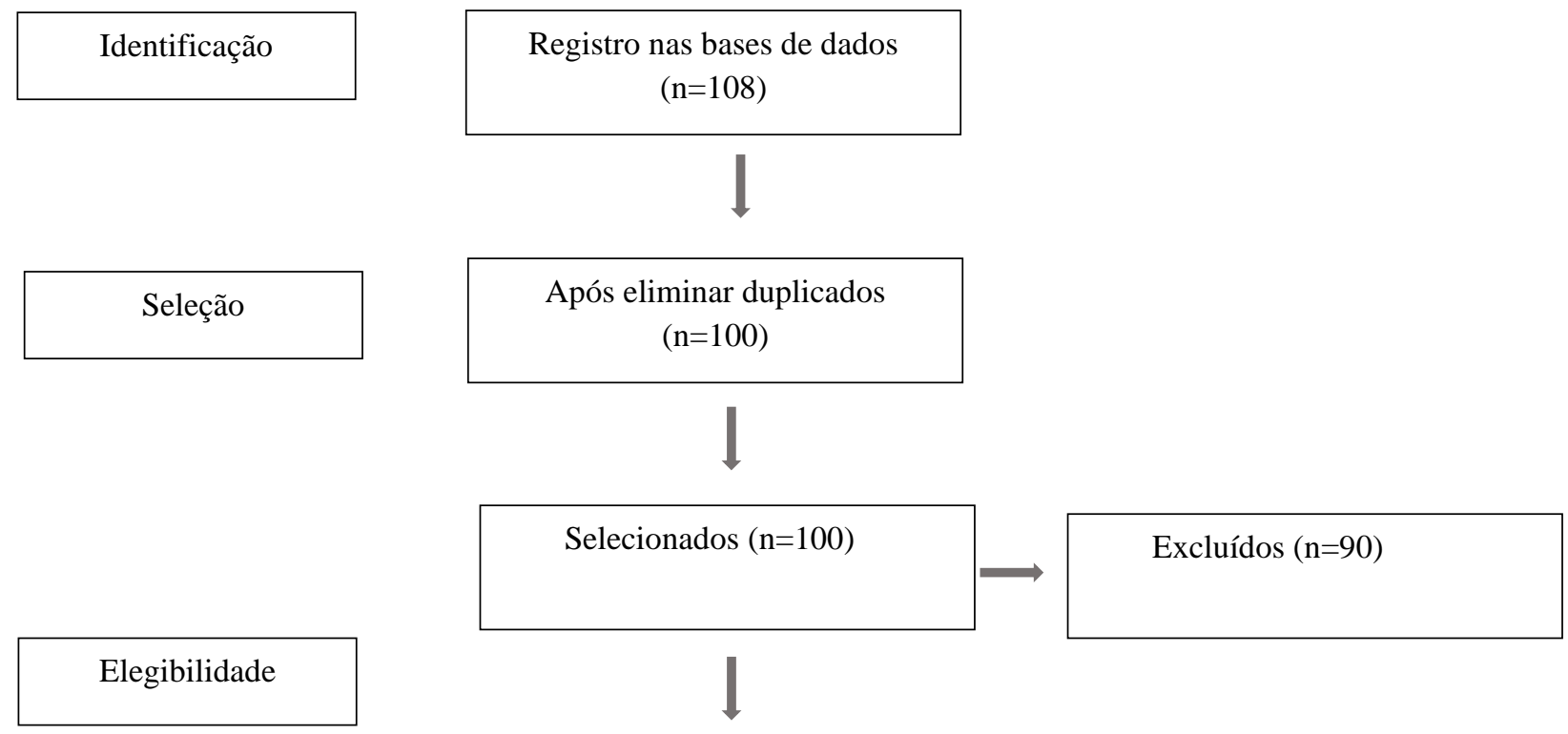

Artigo completo para

elegibilidade $(\mathrm{n}=10)$

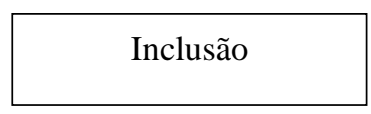

Estudos incluídos em síntese

qualitativa $(\mathrm{n}=10)$

Fonte: Autores.

Entre os artigos, 8 foram eliminados, pois estavam presentes em todas as bases de dados sendo considerado dessa forma duplicado. A leitura dos títulos e dos resumos dos artigos foram os métodos utilizados para inclusão e exclusão, tendo sido eliminados 90 publicações. Ao final da análise alcançou-se a amostra final de 10 estudos primários. Os artigos excluídos não correspondiam durante a leitura de seu título ou resumo ao desfecho esperados nesta revisão ou não se relacionavam ao escopo central dessa pesquisa.

Após elegibilidade dos artigos, eles foram organizados em um quadro (Quadro 1) com extração dos dados baseada na ferramenta Mixed Methods Appraisal Tool (MMAT) (Galvão \& Ricarte, 2019; Oliveira et al., 2021) com a descrição do autor, ano de publicação, periódico, objetivo do estudo, tipo de estudo e resultado. 
Quadro 1. Caracterização dos artigos selecionados por autor, ano de publicação, periódico, objetivo, tipo de estudo e resultados.

\begin{tabular}{|c|c|c|c|c|}
\hline Autor/ano & Periódico & Objetivo & $\begin{array}{l}\text { Tipo } \\
\text { Estudo }\end{array}$ & Resultados \\
\hline $\begin{array}{l}\text { Elhennawy K e } \\
\text { Schwendicke } \\
\text { F. } 2016\end{array}$ & $\begin{array}{l}\text { Journal of } \\
\text { Dentistry } \\
\text { Elsevier Ltd. }\end{array}$ & $\begin{array}{l}\text { Revisar sistematicamente as } \\
\text { modalidades de tratamento para } \\
\text { molares e incisivos afetados por } \mathrm{HMI}\end{array}$ & $\begin{array}{l}\text { Revisão } \\
\text { sistemática }\end{array}$ & $\begin{array}{l}\text { Selantes parecem adequados para molares } \\
\text { HMI com gravidade limitada e / ou } \\
\text { hipersensibilidade. Para casos graves, } \\
\text { restaurações com compósitos ou restaurações } \\
\text { indiretas ou coroas de metal pré-formadas } \\
\text { parecem adequados. Antes do dente extração } \\
\text { como fatores de último recurso, como a } \\
\text { presença de uma má oclusão geral, a idade } \\
\text { dos pacientes e o estado de dentes vizinhos } \\
\text { devem ser considerados. }\end{array}$ \\
\hline $\begin{array}{l}\text { Fragelli, CMB } \\
\text { et al. } 2017\end{array}$ & Braz. oral res. & $\begin{array}{l}\text { Avaliar a sobrevida clínica de selantes } \\
\text { aplicados em primeiros molares } \\
\text { permanentes (PMPs) afetados por } \\
\text { hipomineralização molar-incisivo } \\
\text { (HMI), aos } 18 \text { meses de seguimento. }\end{array}$ & $\begin{array}{l}\text { Estudo clínico } \\
\text { prospectivo }\end{array}$ & $\begin{array}{l}\text { O acompanhamento de } 18 \text { meses indicou que } \\
\text { a sobrevivência dos selantes em PMPs } \\
\text { afetados por HMI não é significativamente } \\
\text { diferente daquela dos PMPs não afetados por } \\
\text { HMI, sugerindo que a aplicação do selante } \\
\text { pode ser uma abordagem eficaz para prevenir } \\
\text { lesões de cárie em HMI- PMPs afetados. }\end{array}$ \\
\hline $\begin{array}{l}\text { Da Cunha } \\
\text { Coelho, ASE } \\
\text { et al., } 2018\end{array}$ & $\begin{array}{l}\text { J Esthet Restor } \\
\text { Dent. } \\
\text { Wiley } \\
\text { Periodicals, } \\
\text { Inc. }\end{array}$ & $\begin{array}{l}\text { Avaliar a eficácia dos tratamentos } \\
\text { aplicados às diferentes formas de } \\
\text { hipomineralização dentária }\end{array}$ & $\begin{array}{l}\text { Revisão } \\
\text { sistemática }\end{array}$ & $\begin{array}{l}\text { Foi possível identificar vários tratamentos } \\
\text { eficazes para dentes com HMI: pastas de } \\
\text { arginina ou vernizes fluoretados) e } \\
\text { clareamento dental e / ou microabrasão do } \\
\text { esmalte. }\end{array}$ \\
\hline $\begin{array}{l}\text { Vieira, LDS et } \\
\text { al. } 2019\end{array}$ & $\begin{array}{l}\text { Medicine } \\
\text { (Baltimore) }\end{array}$ & $\begin{array}{l}\text { Avaliar o efeito clínico da terapia } \\
\text { fotodinâmica antimicrobiana (aPDT) } \\
\text { em dentes permanentes com HMI } \\
\text { grave e com sensibilidade dolorosa, } \\
\text { associada à presença de lesão de cárie. }\end{array}$ & $\begin{array}{l}\text { Ensaio clínico } \\
\text { controlado }\end{array}$ & $\begin{array}{l}\text { Situações de lesões profundas associadas ao } \\
\text { HMI (principalmente com risco de exposição } \\
\text { pulpar) deve-se aplicar a remoção seletiva da } \\
\text { dentina mole, ou seja, a retirada desse tecido } \\
\text { mole apenas das paredes circunvizinhas, } \\
\text { mantendo a dentina mole da parede pulpar. }\end{array}$ \\
\hline $\begin{array}{l}\text { Dhareula, A. et } \\
\text { al. } 2019\end{array}$ & $\begin{array}{l}\text { European } \\
\text { Academy of } \\
\text { Paediatric } \\
\text { Dentistry }\end{array}$ & $\begin{array}{l}\text { O presente ensaio comparou o } \\
\text { desempenho clínico e radiográfico de } \\
36 \text { meses de metal fundido } \\
\text { minimamente invasivo e onlays } \\
\text { indiretos de resina para reabilitação de } \\
\text { primeiros molares permanentes } \\
\text { afetados com HMI grave. }\end{array}$ & $\begin{array}{l}\text { Ensaio clínico } \\
\text { randomizado }\end{array}$ & $\begin{array}{l}\text { Independentemente do tipo de material } \\
\text { usado, as onlays oferecem uma alternativa } \\
\text { restauradora previsível e conservadora para } \\
\text { molares afetados com HMI grave. }\end{array}$ \\
\hline $\begin{array}{l}\text { Linner, } T \text { et al. } \\
2020\end{array}$ & $\begin{array}{l}\text { Internacional } \\
\text { Journal of } \\
\text { paediatric } \\
\text { dentistry }\end{array}$ & $\begin{array}{l}\text { Comparar a longevidade dos } \\
\text { procedimentos de tratamento com } \\
\text { cimento de ionômero de vidro não } \\
\text { invasivo (CIV-R), restaurações de } \\
\text { resina composta, restaurações de } \\
\text { resina e cerâmica convencionais } \\
\text { colocadas em dentes permanentes } \\
\text { com quebra de esmalte devido à } \\
\text { hipomineralização molar-incisivo } \\
\text { (HMI) }\end{array}$ & $\begin{array}{l}\text { Estudo de } \\
\text { coorte } \\
\text { retrospectivo }\end{array}$ & $\begin{array}{l}\text { Restaurações compostas convencionais e } \\
\text { restaurações de cerâmica CAD / CAM } \\
\text { colocadas em dentes afetados por HMI após } \\
\text { o preparo cavitário foram associadas a taxas } \\
\text { de sobrevida de moderadas a altas. Em } \\
\text { contraste, compósito não invasivo e, } \\
\text { especialmente, restaurações em CIV-R sem } \\
\text { preparo, que foram predominantemente } \\
\text { usadas em crianças menos cooperativas, } \\
\text { foram associadas a taxas de sobrevivência } \\
\text { mais baixas, mas podem ajudar a proteger o } \\
\text { tecido duro desintegrado e aumentar a } \\
\text { cooperação dos pacientes até que estejam } \\
\text { maduros o suficiente para lidar com a } \\
\text { implementação de opções de tratamento mais } \\
\text { invasivas, mas de longa duração. }\end{array}$ \\
\hline
\end{tabular}




\begin{tabular}{|c|c|c|c|c|}
\hline $\begin{array}{l}\text { Schraverus MS } \\
\text { et al. } 2021\end{array}$ & Caries Res & $\begin{array}{l}\text { Avaliar o efeito preventivo do } \\
\text { cimento de ionômero de vidro (CIV) } \\
\text { contra cárie dentária e quebra póstero- } \\
\text { posterior (QPP) em molares afetados } \\
\text { por hipomineralização molar incisivo } \\
\text { (HMI). }\end{array}$ & $\begin{array}{l}\text { Ensaio clínico } \\
\text { randomizado }\end{array}$ & $\begin{array}{l}\text { Mostraram que os molares que não foram } \\
\text { selados tinham } 77 \% \text { mais probabilidade de } \\
\text { desenvolver cáries dentárias em comparação } \\
\text { com os molares que receberam uma } \\
\text { aplicação de selante. Também foi } \\
\text { argumentado que a aplicação do selante } \\
\text { ajuda a diminuir a hipersensibilidade } \\
\text { permitindo uma melhor remoção da placa } \\
\text { pelo paciente e, portanto, contribuindo para a } \\
\text { prevenção da cárie. }\end{array}$ \\
\hline $\begin{array}{l}\text { Rolim, TZC et } \\
\text { al. } 2021\end{array}$ & $\begin{array}{l}\text { Clin Oral } \\
\text { Investig }\end{array}$ & $\begin{array}{l}\text { Este ensaio clínico randomizado } \\
\text { avaliou a sobrevivência de } \\
\text { restaurações diretas nos primeiros } \\
\text { molares permanentes (PMPs) com } \\
\text { hipomineralização molar- incisivo } \\
\text { (HMI) e seu impacto na dor dentária } \\
\text { autorreferida e na ansiedade } \\
\text { odontológica. }\end{array}$ & $\begin{array}{l}\text { Ensaio clínico } \\
\text { randomizado }\end{array}$ & $\begin{array}{l}\text { Um adesivo universal pode ser apropriado } \\
\text { para a restauração de dentes afetados por } \\
\text { HMI, e a sobrevida das restaurações pode ser } \\
\text { maior na técnica de condicionamento total, } \\
\text { reduzindo a dor dentária e a ansiedade. }\end{array}$ \\
\hline $\begin{array}{l}\text { Nogueira, } \\
\text { VKC et al. } \\
2021\end{array}$ & $\begin{array}{l}\text { Journal of } \\
\text { Dentistry } \\
\text { Elsevier Ltd. }\end{array}$ & $\begin{array}{l}\text { Integridade estrutural dos dentes } \\
\text { afetados por HMI após o tratamento } \\
\text { com flúor infiltração de verniz ou } \\
\text { resina: um ensaio clínico randomizado } \\
\text { de } 18 \text { meses }\end{array}$ & $\begin{array}{l}\text { Ensaio clínico } \\
\text { randomizado } \\
\text { controlado } \\
\text { simples-cego }\end{array}$ & $\begin{array}{l}\text { A infiltração de resina influenciou } \\
\text { positivamente a manutenção da integridade } \\
\text { estrutural dos dentes afetados por HMI } \\
\text { diminuindo o risco de ruptura do esmalte ao } \\
\text { longo de } 18 \text { meses de acompanhamento. }\end{array}$ \\
\hline $\begin{array}{l}\text { De Farias, AL, } \\
\text { et al. } 2021\end{array}$ & $\begin{array}{l}\text { Int J Paediatr } \\
\text { Dent }\end{array}$ & $\begin{array}{l}\text { Avaliar a sobrevivência de } \\
\text { restaurações com coroa de aço } \\
\text { inoxidável (CAI) ou resina composta } \\
\text { (RC) em primeiros molares } \\
\text { permanentes afetados por HMI por } 24 \\
\text { meses. }\end{array}$ & $\begin{array}{l}\text { Estudo de } \\
\text { coorte } \\
\text { retrospectivo }\end{array}$ & $\begin{array}{l}\text { Em molares com HMI com necessidade de } \\
\text { tratamento restaurador, CAI teve uma } \\
\text { significativa maior taxa de sobrevivência do } \\
\text { que RC ao longo de } 24 \text { meses. }\end{array}$ \\
\hline
\end{tabular}

Fonte: Autores.

\section{Discussão}

Após análise dos 10 artigos notou-se que as modalidades de tratamento para os dentes afetados por HMI variam amplamente, dependendo da gravidade, e da presença de fatores complicadores, como hipersensibilidade, higiene oral e cooperação do paciente. Segundo Fragelli et al. (2021) os dentes afetados por HMI requerem cuidados preventivos, devido a um alto risco de fraturas pós-eruptivas resultantes das forças mastigatórias e desafios acidogênicos da cavidade bucal. Uma vez ocorrida a fratura, o esmalte e / ou dentina são expostos, resultando em sensibilidade ao frio, calor e escovação e, possivelmente, levando a maior acúmulo de placa e desenvolvimento de lesões cariosas. O tratamento com selante é uma alternativa eficaz para prevenção da doença cárie.

O estudo de Schraverus et al. (2021), mostrou que os molares que não foram selados tinham 77\% maior probabilidade de desenvolver lesões de cárie dental em comparação com os molares que receberam uma aplicação de selante de ionômero de vidro (CIV). Esse efeito protetor é devido à atuação do selante como uma barreira mecânica, isolando fisicamente as fossas e fissuras do meio bucal. Além disso, uma das principais vantagens do CIV é a liberação de flúor, que está associado à prevenção da doença cárie. Outra característica positiva oferecida é a diminuição da hipersensibilidade, permitindo uma melhor higienização oral do paciente e, portanto, contribuindo para a prevenção da cárie.

O esmalte hipomineralizado sofre alterações químicas, mecânicas e morfológicas resultando em uma difícil adesão desse selante, sendo que a adesão é um fator chave para o sucesso desse tratamento. Embora essas alterações possam resultar em complicações para a adesão de materiais odontológicos, segundo Fragelli et al. (2017), o acompanhamento de 18 meses indicou que a sobrevivência dos selantes em molares afetados por HMI não é significativamente diferente daquela dos não 
afetados por HMI, sugerindo que a aplicação do selante é uma abordagem eficaz para prevenção de lesões de cárie em molares afetados por HMI.

Elhennawya e Schwendicke (2016), afirmam também que em primeiro lugar, a hipersensibilidade precisa ser tratada. Eles mostram que a caseína fosfato de cálcio fosfopeptídeo amorfo (CFC-ACP) demonstrou ter um efeito remineralizador e dessensibilizante em dentes com HMI, pois produz uma solução supersaturada estável de cálcio e fosfato na superfície do esmalte. Em oposição, Schraverus et al (2021) afirmam que não há diferença significativa do efeito dessensibilizante do CFC ACP com ou sem flúor e que os dados disponíveis ainda são insuficientes para apoiar a aplicação de flúor para HMI. No entanto, relatam que os selantes de fissuras podem ter alguns benefícios para HMI leve em molares, pois auxiliam ainda mais na prevenção de lesões de cárie dental e quebra do esmalte.

Nogueira et al. (2021) realizaram um ensaio clínico randomizado, no qual avaliaram a realização da terapia de verniz fluoretado (VF) e uma técnica de infiltração de resina em lesões HMI amarelas e / ou marrons por 18 meses. A terapia de infiltração de resina (TIR) é baseada na penetração de uma resina à base de TEGDMA de baixa viscosidade já que esta promove a obliteração das porosidades. Os resultados mostraram que a taxa de quebra pós-eruptiva do esmalte (QPEE) em TIR foi significativamente menor do que com verniz fluoretado.

Vieira et al. (2019) afirmam que quando os dentes já estão afetados por lesões de cárie, o tratamento deve respeitar os princípios da mínima intervenção. Deve ser realizada a remoção da dentina infectada/amolecida apenas das paredes circundantes, mantendo esse tecido nas proximidades da parede pulpar. Logo após essa remoção seletiva é realizada uma terapia fotodinâmica antimicrobiana, com o uso do gel PapacárieMblue, modificado com a adição de azul de metileno como fotossensibilizador, em conjunto com o laser de baixa potência para dessensibilização e descontaminação das cavidades.

O desafio de adesão ao esmalte hipomineralizado é relatado também por Rolim et al. (2021), sendo uma dificuldade em realizar as restaurações diretas, pois o esmalte hipomineralizado tem menos conteúdo mineral, alto teor proteico, e menor resistência relacionada à microdureza, em relação às forças mastigatórias. Estudos anteriores recomendam a remoção total do esmalte hipomineralizado até que um esmalte sólido seja obtido, melhorando a resistência de união de materiais restauradores a superfície dentária. O trabalho de Rolim et al (2021) demonstra que mesmo o esmalte acometido por HMI, ainda que sem danos aparentes possui alterações em sua estrutura quando comparada com um esmalte sadio, portanto, comprometendo a adesão dos materiais restauradores mesmo após a remoção extensiva do tecido dentário. Desse modo, eles defendem a remoção seletiva do esmalte hipomineralizado, baseado na prática da odontologia de mínima intervenção.

Restaurações indiretas também são uma opção de tratamento como demonstrado no ensaio clínico randomizado de Dhareula et al. (2021) que relatam que coroas parciais do tipo onlay são viáveis e fornecem uma opção clínica conservadora para molares afetados por HMI mas também podem ser substituídas por coroas totais, se necessário. O desempenho satisfatório de onlays com mais de 36 meses de acompanhamento demonstraram uma alternativa estética ao metal fundido tradicional. Farias et al. (2021) também mostraram que em primeiros molares permanentes com HMI, tratados com restaurações indiretas ainda que de aço inoxidável tem maior eficácia terapêutica do que restaurações em resina composta, por um período de 24 meses.

Segundo Elhennawya e Schwendicke (2016), nos casos de defeitos graves em molares algumas condutas são propostas e variam de opções conservadoras como as restaurações em resina composta, evoluindo para coroas de metal pré-formadas ou restaurações indiretas quando a estrutura dental não possui mais remanescente suficiente, do ponto de vista de paredes circundantes para receber restaurações diretas e culminando na última opção restauradora e a mais mutiladora que se trata da exodontia, que deve ser indicada na impossibilidade dos tratamentos citados anteriormente e deve ser realizada associada a um planejamento odontológico integral e integrado, seguido de terapia ortodôntica subsequente para devolução da funcionalidade e estética dos arcos dentais. 


\section{Conclusão}

Diante do exposto que diversas abordagens clínicas são propostas na literatura atual no intuito de controlar os três principais desafios terapêuticos em hipomineralização molar-incisivo: as fraturas dentais, a hipersensibilidade dentinária e a dificuldade de adesão. Os selantes parecem ser uma alternativa para evitar fraturas e também o desenvolvimento de lesões de cárie dental. Para o tratamento da hipersensibilidade dentinária terapias com laser, isoladas ou associadas a outras modalidades de tratamento como a infiltração de resina parecem ser eficazes, embora a literatura ainda não seja uníssona sobre estas abordagens. Os materiais adesivos podem ser utilizados, embora as alterações físicas e químicas nos tecidos dentais interfiram em sua eficácia e por vezes restaurações indiretas podem ser utilizadas com bons resultados clínicos. Mais estudos, com metodologias robustas e amostras bem selecionadas são necessários no intuito de determinar protocolos clínicos homogêneos e eficazes para o tratamento de hipomineralização molar-incisivo.

\section{Referências}

Alvarez Ochoa, D., Robles Contreras, I., Díaz Meléndez, J., \& Sandoval Vidal, P. (2017). Abordaje Terapéutico de la Hipomineralización Molar-Incisal. Revisión Narrativa. International journal of odontostomatology, 11(3), 247-251. http://dx.doi.org/10.4067/S0718-381X2017000300247.

Bezamat, M., Souza, J. F., Silva, F., Corrêa, E. G., Fatturi, A. L., Brancher, J. A., Carvalho, F. M., Cavallari, T., Bertolazo, L., Machado-Souza, C., Koruyucu, M., Bayram, M., Racic, A., Harrison, B. M., Sweat, Y. Y., Letra, A., Studen-Pavlovich, D., Seymen, F., Amendt, B., Werneck, R. I., ... Vieira, A. R. (2021). Gene-environment interaction in molar-incisor hypomineralization. PloS one, 16(1), e0241898. https://doi.org/10.1371/journal.pone.0241898.

Cunha, L. S., Lima, C. C., Braga, L. L. A., Andrade, C. M. de O., Caixeta, D. A. F., \& Dietrich, L. (2020). Hipomineralização Molar-Incisivo em dentes permanentes: revisão de literatura. Research, Society and Development, 9(11), e889119653. https://doi.org/10.33448/rsd-v9i11.9653

Cunha Coelho, A., Mata, P., Lino, C. A., Macho, V., Areias, C., Norton, A., \& Augusto, A. (2019). Dental hypomineralization treatment: A systematic review. Journal of esthetic and restorative dentistry: official publication of the American Academy of Esthetic Dentistry. 31(1), 26-39. https://doi.org/10.1111/jerd.12420

Silva, A. R. S. F., Moraes, G. D. M., Guedes, P. D. F., Koga, R. S., \& Carlos, A. M. P. (2020). Tratamento de hipomineralização molar-incisivo em odontopediatria: revisão de literatura. Brazilian Journal of Health Review, 3(6), 16789-16801.

Farias, A. L., Rojas-Gualdrón, D. F., Mejía, J. D., Bussaneli, D. G., Santos-Pinto, L., Restrepo, M. (2021) Survival of stainless steel crowns and composite resin restorations in molars affected by molar-incisor hypomineralization (HMI). Int J Paediatr Dent. DOI: 10.1111/ipd.12849.

Dhareula, A., Goyal, A., Gauba, K., Bhatia, S. K., Kapur, A., \& Bhandari, S. (2019). A clinical and radiographic investigation comparing the efficacy of cast metal and indirect resin onlays in rehabilitation of permanent first molars affected with severe molar incisor hypomineralisation (HMI): a 36-month randomised controlled clinical trial. European archives of paediatric dentistry: official journal of the European Academy of Paediatric Dentistry, 20(5), 489500. https://doi.org/10.1007/s40368-019-00430-y

Elhennawy, K., \& Schwendicke, F. (2016). Managing molar-incisor hypomineralization: A systematic review. Journal of dentistry, 55, 16-24. https://doi.org/10.1016/j.jdent.2016.09.012

Farias, L., Laureano, I. C. C., Alencar, C. R. B. de, \& Cavalcanti, A. L. (2018). Hipomineralização molar-incisivo: etiologia, características clínicas e tratamento. Revista De Ciências Médicas E Biológicas, 17(2), 211-219. https://doi.org/10.9771/cmbio.v17i2.27435

Folayan, M. O., Chukwumah, N. M., Popoola, B. O. et al. (2018). Developmental defects of the enamel and its impact on the oral health quality of life of children resident in Southwest Nigeria. BMC Oral Health 18, 160. https://doi.org/10.1186/s12903-018-0622-3

Fragelli, C., Souza, J. F., Bussaneli, D. G., Jeremias, F., Santos-Pinto, L. D., \& Cordeiro, R. (2017). Survival of sealants in molars affected by molar-incisor hypomineralization: 18-month follow-up. Brazilian oral research, 31, e30. https://doi.org/10.1590/1807-3107BOR-2017.vol31.0030

Galvão, M. C. B., \& Ricarte, I. L. M. (2019). Revisão sistemática da literatura: conceituação, produção e publicação. Logeion: Filosofia Da Informação, 6(1),57-73. https://doi.org/10.21728/logeion.2019v6n1.

Lacruz, R. S., Habelitz, S., Wright, J. T., \& Paine, M. L. (2017). Dental enamel formation and implications for oral health and disease. Physiological reviews, 97(3), 939-993. https://doi.org/10.1152/physrev.00030.2016

Linner, T., Khazaei, Y., Bücher, K., Pfisterer, J., Hickel, R., \& Kühnisch, J. (2020). Comparison of four different treatment strategies in teeth with molarincisor hypomineralization-related enamel breakdown-A retrospective cohort study. International journal of paediatric dentistry, 30(5), 597-606. https://doi.org/10.1111/ipd.12636

López Jordi, M. D. C., Cortese, S. G., Álvarez, L., Salveraglio, I., Ortolani, A. M., \& Biondi, A. M. (2014).Comparación de la prevalencia de hipomineralización molar incisiva em niños con diferente cobertura asistencial en las ciudades de Buenos Aires (Argentina) y Montevideo (Uruguay). Saúde Coletiva Lanús, 10 (2), 243-251. 
Research, Society and Development, v. 10, n. 13, e561101321605, 2021

(CC BY 4.0) | ISSN 2525-3409 | DOI: http://dx.doi.org/10.33448/rsd-v10i13.21605

Nogueira, V., Mendes Soares, I. P., Fragelli, C., Boldieri, T., Manton, D. J., Bussaneli, D. G., \& Cordeiro, R. (2021). Structural integrity of HMI-affected teeth after treatment with fluoride varnish or resin infiltration: An 18-Month randomized clinical trial. Journal of dentistry, 105, 103570. https://doi.org/10.1016/j.jdent.2020.103570

Oliveira, J.L.C., Magalhães, A.M.M., Matsuda, L.M., Santos, J.L.G., Souto, R.Q., Riboldi, C.O., Ross, R. Mixed methods appraisal tool: strengthening the methodological rigor of mixed methods research studies in nursing. Texto \& Contexto - Enfermagem [online]. (30). <https://doi.org/10.1590/1980-265X-TCE2020-0603>. Epub 27 Ago 2021. ISSN 1980-265X. https://doi.org/10.1590/1980-265X-TCE-2020-0603.

Rolim, T., da Costa, T., Wambier, L. M., Chibinski, A. C., Wambier, D. S., da Silva Assunção, L. R., de Menezes, J., \& Feltrin-Souza, J. (2021). Adhesive restoration of molars affected by molar incisor hypomineralization: a randomized clinical trial. Clinical oral investigations, 25(3), 1513-1524. https://doi.org/10.1007/s00784-020-03459-2

Schraverus, M. S., Olegário, I. C., Bonifácio, C. C., González, A., Pedroza, M., \& Hesse, D. (2021). Glass Ionomer Sealants Can Prevent Dental Caries but Cannot Prevent Posteruptive Breakdown on Molars Affected by Molar Incisor Hypomineralization: One-Year Results of a Randomized Clinical Trial. Caries research, 55(4), 301-309. https://doi.org/10.1159/000516266

Schwendicke, F., Elhennawy, K., Reda, S., Bekes, K., Manton, D. J., \& Krois, J. (2018). Global burden of molar incisor hypomineralization. Journal of dentistry, 68, 10-18. https://doi.org/10.1016/j.jdent.2017.12.002

Smith CEL, Poulter JA, Antanaviciute A, Kirkham J, Brookes SJ, Inglehearn CF and Mighell AJ (2017) Amelogenesis Imperfecta; Genes, Proteins, and Pathways. Front. Physiol. 8:435. doi: 10.3389/fphys.2017.00435

Vieira, L., Paschoal, M., de Barros Motta, P., Ferri, E. P., Ribeiro, C., Dos Santos-Pinto, L., Motta, L. J., Gonçalves, M., Horliana, A., Fernandes, K., Ferrari, R., Deana, A. M., \& Bussadori, S. K. (2019). Antimicrobial photodynamic therapy on teeth with molar incisor hypomineralization-controlled clinical trial. Medicine, 98(39), e17355. https://doi.org/10.1097/MD.0000000000017355 